\title{
Experiences on building a distributed computing platform prototype for telecom network and service management
}

\author{
Rahkila, S., Stenberg, S.
}

Nokia Research Center, Distributed Computing Platforms, P.O.Box 422, 00045 Nokia Group, Finland, phone: +358-9-43761 sakari.rahkila@research.nokia.com susanne.stenberg@research.nokia.com

\begin{abstract}
Open object-oriented distributed computing will be essential for the telecommunications business in the future. The most promising approach for the object-oriented distributed computing is the Common Object Request Broker Architecture (CORBA) technology standardized by Object Management Group (OMG). CORBA forms the foundation for building applications constructed from distributed objects and for interoperability between applications in homogeneous and heterogeneous computing environments.

The World Wide Web has grown to become one of the most popular services on the Internet. An integration of the Web and CORBA enable the benefits of distributed object computing while using existing Web resources.

The integration of the two recognized network management protocol standards CMIP and SNMP, and CORBA technology, allows management applications to take advantage of distributed object computing as well as the standardized network management protocols.

Nokia Research Center has launched a project to build a platform prototype on an object-oriented distribution infrastructure. The purpose of the prototype is to provide a framework which supports the creation, management and invocation of distributed telecom services.
\end{abstract}

\section{Keywords}

Network Management, Service Management, World Wide Web, CORBA 


\section{INTRODUCTION}

Telecommunications networks are continuously growing in scale and complexity, and the number of equipment and services they provide is increasing. Integrated, distributed management of heterogeneous networks and services is increasingly important. There is thus a need for a consistent approach to integrate management solutions. Additionally it would be desirable to use off-the-shelf (buy vs. build) components and to leverage existing investments by integration of 'legacy' management applications. Distribution in management applications is needed for the scalability and for the cost/performance benefits.

The CORBA [1] technology has gained credit solving distribution, interface and integration problems. However, CORBA does not provide a network management architecture; it provides a distributed object computing architecture.

In the telecommunications industry the Telecommunications Management Network (TMN) [2] paradigm is widely established. In the Internet community, on the other hand, SNMP [3] based network management has gained widespread acceptance due to its simplicity of implementation. Thus, TMN and Internet management will coexist far in the future.

Currently the Web based technology is changing and evolving very rapidly, and has attracted a lot of attention also for network management solutions. However, the Web development has been very closely based on the use of the Hypertext Markup Language (HTML) and the Common Gateway Interface (CGI), which are very limited in functionality. Therefore, new approaches are needed to support utilization of resources at the client's end and distribution of services over the network. This also includes the use of 'mobile code', such as JavaSoft's Java for easy integration of existing Web browser and the object-oriented CORBA technology.

Below we present the TMN, SNMP, CORBA and Web concepts, and describe the effort in the Nokia Distributed Computing Platform (DCP) prototype project to integrate these technologies.

\section{INTRODUCTION OF TECHNOLOGIES}

\subsection{Network Management}

The goal of TMN is to enhance interoperability of management software and to provide an architecture for management systems. A TMN is a logically distinct network from the telecommunications network that it manages. It interfaces with the telecommunications network at several different points to send/receive information to/from it and to control its operations.

The TMN information architecture is based on an object-oriented approach and the agent/manager concepts that underlie the Open Systems Interconnection (OSI) systems management [4]. For a specific management association, the management processes involved will take on one of two possible roles: a manager and an agent. A managed object is the OSI abstract view of a logical or physical system resource to be managed. All management exchanges between a manager and an agent are expressed 
in terms of a consistent set of management operations and notifications. A Managed Object is the OSI abstract view of a logical or physical system resource to be managed. These operations are realized through the use of the CMIS [5] and the CMIP [6].

SNMP is a protocol suite developed for the management of the Internet. SNMP was designed to be an application level protocol that is a part of TCP/IP protocol suite.

The SNMP framework is based on the principle of minimally simple agents and complex managers. Internet uses the term network element to describe any object that is managed. The network element consists of the managed entity and the managed entity's agent [7]. The SNMP structure of information uses simple two-dimensional tables as it's basic containment structure for managed objects.

\subsection{CORBA}

The Object Management Architecture includes the Object Request Broker (ORB), Application Interfaces, Common Facilities, Object Services, and Domain Interfaces.

Application Interfaces are specific to end-user applications, they are not defined in the OMA architecture.

Domain Interfaces are vertical application domain-specific interfaces, e.g. domain interfaces for telecommunication applications.

Common Facilities [8] are horizontal end-user-oriented facilities applicable to most application domains (e.g. Distributed Document Component Facility).

Object Services [9] is a collection of services with object interfaces, which provides basic functions for all objects to be shared by all applications. The Object Services are intended to be modular so that clients in the client/server environment are free to use as many or as few as necessary to accomplish a task.

The Object Request Broker provides the basic mechanism for transparently making requests to - and receiving responses from - objects located locally or remotely. The ORB supplies delivery services, activation and deactivation of remote objects, method invocation, parameter encoding, synchronization, and exception handling. For interoperability between ORBs, CORBA 2.0 specifies a mandatory message format, called General Inter-ORB Protocol (GIOP), which can be hosted on any network transport in theory, however, TCP/IP is mandatory. Hosted on TCP/IP the protocol is called Internet Inter-ORB Protocol (IIOP).

The CORBA Interface Definition Language is used to define the interface to a CORBA object. IDL is programming language independent. It is mapped to the implementation language, i.e. the programming language in which the object implementation code is written. IDL compiler output binds clients and object implementations to the ORB.

\subsection{Web and Java}

The Web is largely founded on the use of two Internet standards, HTTP and HTML. The HTTP is an application-level protocol, which defines both message formats and a mapping to TCP/IP. HTTP is a stateless protocol, in which headers are transferred as (ASCII) text. The HTML defines the data formats for creating documents (pages) that are available via servers. HTML is not really a programming language. It specifies the 
use of tags to code information, which tells the Web browser how to display the documents' text elements. The documents are identified by an Uniform Resource Locator (URL), which can point to a static document, i.e. a file, or to a dynamic document. A dynamic document is one which is generated by a program which is identified by the URL. This mechanism is provided by CGI. The CGI is a standard for external gateway programs to interface with information servers such as HTTP servers.

The Web architecture has scalability problems, e.g. all interactions to a remote system go through one HTTP server. Another problem is the client interactions with the HTTP server, in which the HTTP protocol opens and closes a new connection for each request.

The integration of the Web and CORBA alleviates some of the scalability problems in the Web architecture. The use of CORBA removes the Web server as a single focal point for all client requests, and compared to HTTP, the IIOP is a far more promising protocol for reaching resources over TCP/IP. The Web and CORBA applications can be integrated using a gateway approach. The definition of a CORBA IDL mapping for HTTP enables interoperability between ORB/HTTP domains. Another simple approach is to use the CGI gateway. A CGI program can be associated with a CORBA client.

Java is an object-oriented programming language created by JavaSoft. Java is interpreted and features automatic garbage collection. The Java compiler generates bytecode instructions which are executed using interpreters provided for a variety of platforms. Thus, Java code is considered architecture-neutral. If additional performance is required, the Java bytecode can be translated into machine code for a particular CPU.

Applets are Java programs that follow a set a conventions that allow them to run within a Java enabled browser, i.e. a browser including the Java interpreter. A Java applet is made up of one or more Java objects. Java applets are embeddable into HTML documents, which means they can be down-loaded on-demand from a server and executed in the Web browser. In a sense Java represents mobile code.

For Web development, Java applets provide far better facilities than HTML forms. The applets are more dynamic and are reusable.

The natural way to integrate Java and CORBA is to provide a Java mapping from CORBA IDL. This allows applets to appear as CORBA objects. A Java and CORBA integration supporting the Web is to provide an IIOP package written in Java. This effectively extends the Web browser to support IIOP.

\subsection{Integration of Technologies}

The Joint Inter-Domain Management (JIDM) working group sponsored by X/Open and Network Management Forum (NMF) is seeking to enable inter-operability between CMIP, SNMP, and CORBA. JIDM concentrates on CMIP/CORBA and SNMP/CORBA inter-working.

The NMF has produced specifications that enable interworking between SNMP and CMIP environments. The SNMP/CMIP interworking package consists of five 
specifications. Three of the specifications address the relationships among object definitions in the two models, e.g., the translation of the Internet MIBs to GDMO and vice versa.

As stated in the mission statement of the OMG Telecommunications Domain Task Force, it provides a forum for the exploration, specification, and application of object technology within the telecommunications industry. The Task Force has outlined an architecture for a CORBA-based telecommunication network management system, using ORBs to facilitate the monitoring and control of telecommunications network elements, networks and services.

\section{NOKIA DCP PROTOTYPE}

\subsection{Overview}

The purpose of the Nokia Distributed Computing Platform prototype is to provide an extensive framework which supports the creation, management and invocation of distributed telecom services. The framework is built on an object-oriented 'software bus' that facilitates both service development and service integration. The technology providing the software bus is CORBA. Objects form the components of all services, in this framework which integrates the technologies described above (Figure 1 ).

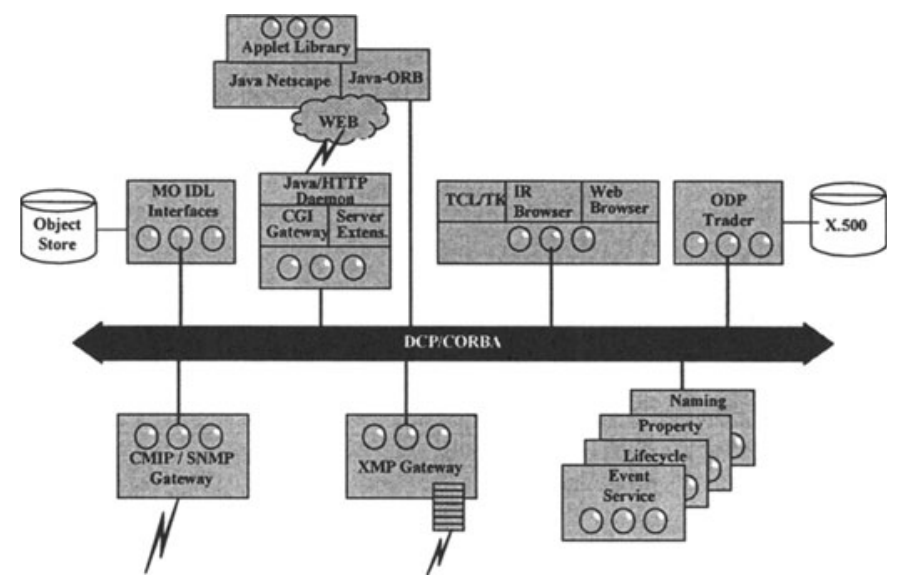

Figure 1 Nokia Distributed Computing Platform Prototype.

The following chapters describes the various parts of the DCP prototype.

\subsection{GDMO/ASN.1 to IDL Compiler}

The DCP prototype includes implementation of GDMO/ASN.1 to IDL compilers in accordance with the JIDM Specification Translation and related class libraries. The 
compilers utilize a persistent metadata repository for storing the knowledge about the GDMO and IDL definitions. The metadata repository stores the information about the GDMO objects using the Management Knowledge Management [10] metadata object definitions. The metadata repository uses an object database for persistence.

Tools for browsing the persistent metadata repository are also produced. These tools include facilities to browse the GDMO metadata using a Web browser such as Netscape or Mosaic and UNIX shell invocation using Tcl/Tk windows.

\subsection{String PDUs}

It is possible to represent all the structures (syntax) used to build CMIS(P) messages using a string representation. This allows the building of management entities, which send and receive string messages. The approach used in the DCP prototype follows the one outlined in [11].

The string API is based on ASN.1 and hence allows any syntax defined in ASN.1 to be passed. Each message is built as a string which includes three basic pieces of information. These are the ASN.1 module name, the ASN.1 type name, and a string containing the value.

The DCP prototype contains a string PDU package which is used for building, parsing and passing CMIP string PDUs using CORBA as transport mechanism. The architecture of the package can be divided into three separate areas: ASN.1 data type classes, CMIP data type classes and builder classes. The ASN.1 classes are used to represent the basic ASN.1 data types such as Integer, Real etc. The CMIP classes represent the CMIP datatypes. These classes use the ASN.1 classes to compose a more complex data types. The builder classes are used to create CMIP String PDUs.

\subsection{CMIP/SNMP Gateway}

The CMIP/SNMP gateway enables management of SNMP devices by using CMIS services. The gateway supports sending and receiving of CMIP and SNMP protocol events and timer events, and dispatches methods to managed objects also residing in the gateway.

The managed objects of the OSI/Internet management gateway communicate with Internet agents by using the SNMP protocol to carry out the operations invoked through their CORBA IDL interfaces. The managed objects derived from base classes in the DCP prototype represent the NMF translation of Internet MIBs to ISO/CCITT GDMO MIBs.

The naming service of the DCP prototype is used by the gateway to register managed objects and to enable scoping. The X.500 names-library allows conversion of X.500 names to the CORBA names. The gateway uses the CORBA Life Cycle Service of the DCP prototype to create a managed object corresponding to the managed-object class (object identifier) received as a parameter of CMIS M-CREATE request. CMIP protocol messages are received and sent as string PDUs over CORBA, as described in the previous chapter.

The stateless approach used in the gateway does not maintain the Internet MIB's data. Instead, for each received OSI management service request, the gateway 
generates one or more Internet management service requests (i.e. SNMP requests) to the Internet agent in order to achieve the function of the OSI management service request. A problem with this approach is that certain OSI management notifications which result from a change of state in the MIB cannot be emulated, since many such changes don't cause the Internet agent to send a trap, and a comparison of states in the gateway is impossible because the Internet MIB data is not replicated.

The stateful approach would require the gateway to replicate the Internet MIB locally, and to send periodic requests to SNMP agents to keep the replicated MIB current. The gateway would then try to fulfill each incoming CMIS request by using locally-replicated MIB data, instead of sending appropriate requests to the SNMP agent. The stateful approach could usually provide better response time, but has the drawback that the data retrieved might not be current. The poll frequency used to update the locally-replicated MIB has a significant effect on the accuracy of the response.

The stateless approach was selected because it is far less complex and it is also the approach used in the ISO/CCITT and Internet Management Coexistence (IIMC) documents of NMF.

\subsection{Nokia XMP++}

The Nokia XMP++ [15] classes enable building of C++ based applications on top of the X/Open XMP API [16]. The XMP API provides an industry-standard API to CMIP and SNMP management protocols. A new version of XMP++, having a more generic nature, has been integrated into the DCP prototype. This XMP++ gateway allows building of OSI/CORBA gateway applications.

The XMP API library implementation, which has been used, is the one provided by the HP OpenView Distributed Management (OVDM) platform.

There are three interface layers in $\mathrm{XMP}++$, represented by three groups of classes: netHandle, xmpPlat form, and xmpWkSpace and xmpSession.

The xmpWkSpace and xmpSession classes interface directly with the XMP API; respectively they correspond to the XMP API Workspace and Session concepts. The xmpPlatform class replaces the XMP API Workspace and Session concepts with a single management connection concept. The netHandle and its derived classes are the interface classes for the network management applications to access the network. The netHandle class uses the xmpPlatform class. It also uses the event handle class (Reactor class) from the ADAPTIVE Communication Environment (ACE) library V3.3 [17] for receiving messages from the network and for catching signals from the system.

The XMP++ gateway provides a conversion between the CMIP protocol messages which are received and sent as string PDUs over CORBA, and the information of the XMP++ interface.

The class model of the gateway is based on a MIT actor-dispatcher design [18]. The dispatcher provides for demultiplexing of timer events, signal events and I/O events received through communication channels. It defines an interface for 
registering actors for certain events, and dispatches the incoming messages to the preregistered actors.

The actors are derived from CORBA objects. The actor (base) class declares virtual methods for handling different events that it can register for. The derived actor classes implement the event handling in a specific manner. The proxy actors represent managed objects and take care of the mapping of operations.

The DCP_Gdmo_Disp accepts incoming messages, and forwards them to the appropriate XMP_Proxy_Act (Figure 2 ).

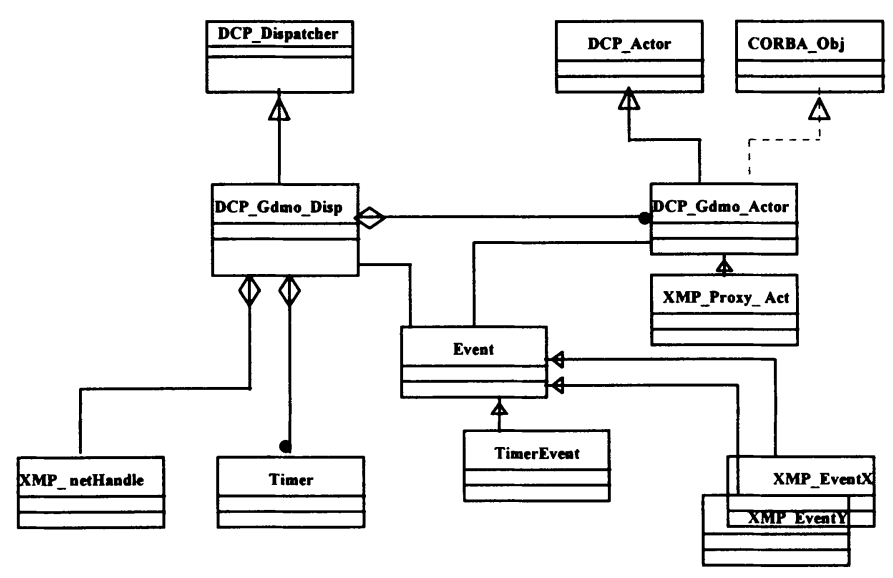

Figure 2 Actor class framework.

The XMP_Proxy_Act performs the conversion and calls the DCP_Gdmo_Disp which sends the message to the network using the XMP_netHandle class. The DCP_Gdmo_Disp keeps track of pending operations and supports e.g. retries.

The Name Service finds the desired CORBA object reference to the XMP_Proxy_Act based on OSI distinguished names.

\subsection{DCP Prototype Services}

The Naming Service is the principal mechanism for objects in the DCP prototype to locate other objects. The COSS Naming Service defines operations how a client can get an object reference by a name. The Naming Service is designated to be built on top of existing name and directory services such as ISO X.500 [12]. Through the use of a names-library, name manipulation is simplified and names can be made representationindependent thus allowing their representation to evolve without requiring client changes. The DCP prototype contains an implementation of a specially designed X.500 names-library allows conversion of X.500 names to the CORBA names.

The concept of a Trading Service [13] provides a mechanism for dynamically finding services. The object which realizes the Trading Service is called trader. A trader is an object that enables clients to find information about suitable services and servers. Service providers can advertise or export services to a trader. Such 
advertisements are known as Service Offers. They describe a service using a set of service properties (name, value, mode triples). The service provider or object acting on behalf of the service provider is called an exporter. Potential service users, importers, can import from the trader, which means obtaining information on available services and their accessibility. The trader tries to match the importer's request against its Service Offers. After a successful match, the importer can interact with the service provider.

Traders can share information about Service Offers among each other. This is realized by a concept known as federation of traders. That is, traders can act recursively as clients to other traders.

The Property Service [14] provides a set of interfaces for dynamically manipulating the properties of objects. A CORBA object supports an interface which consists of operations and attributes. The interface is defined in IDL. Properties are intended to be the dynamic equivalent of CORBA attributes.

The Property Service defines operations to create and manipulate sets of namevalue pairs or name-value-mode tuples. The names are simple IDL strings and the values are IDL anys. The use of type any is significant in that it allows a property service implementation to deal with any value that can be represented in the IDL type system. As with CORBA attributes, clients can get and set property values. However, with properties, clients can also dynamically create and delete properties associated with an object. In addition, clients can create and manipulate properties and their characteristics, such as the property mode. For example, a management application can create an expire date property for a particular service and set the mode of property to be fixed read-only. This means that other clients can read the property (and use the service if it is in use), but can not write or delete the expire date.

The Life Cycle Services defines services and conventions for creating, deleting, copying and moving distributed objects.

To create a new object, a client must find a factory object, which is an object that knows how to instantiate an object of the desired kind. The Life Cycle service defines a GenericFactory interface, which supports a general create_object operation. The LifeCycleObject interface has operations to move, copy and remove objects. The FactoryFinder defines an interface for finding factories.

The DCP prototype includes an implementation of a generic factory with GenericFactory interface as defined in COSS [9]. The job of the generic factory is to match the creation criteria specified by clients with offers of implementation specific factories which actually assembles the resources for objects. Objects of the DCP prototype support the LifeCycleObject interface.

\subsection{Web User Interface}

Web based user interfaces offer some benefits for network management applications. Web browsers provide for cost-benefits, the user interfaces are easy to use, and enables mobile management in that remote access only requires access to the Internet and a Web browser [19]. 
Java applet based UIs allow new versions of the user interfaces to be deployed faster, as they are downloaded from the server. The DCP prototype provides a Java applet library for accessing the distributed services provided by the platform. This includes a set of applets, which provide a user interface to the network management operations. The set contains applets for manipulating tree structures, e.g. MIBs, naming trees etc. The Java applets communicate with the DCP prototype both trough the HTTP-IIOP gateway described below and directly by IIOP using a commercial Java-ORB implementation.

\subsection{HTTP - IIOP Gateway}

The CORBA IDL mapping of the HTTP produced by ANSA treats HTTP as a set of methods with arguments and results. This mapping has been used to provide gateways that can convert between HTTP and CORBA IIOP representations. Interoperability between the DCP prototype and the Web is achieved by using the HTTP-IIOP (H2I) and IIOP-HTTP $(\mathrm{I} 2 \mathrm{H})$ gateways from ANSA. This allows Web clients to use objects (clients) developed on the DCP prototype.

The IIOP-HTTP gateways between the DCP prototype and the Web require a trader to locate new services and to map between URLs and CORBA object references for the services accessible through the platform.

For future development of the DCP prototype the HTTP-IIOP and IIOP-HTTP gateways will not be used, since using Java-ORBs provides a much more natural integration of the Web and CORBA based services.

\section{CONCLUSIONS AND FUTURE ENHANCEMENTS}

We believe that CORBA technology has the potential to become the basis for a paradigm shift from centralised management to distributed management of networks and services. We also believe that future management solutions benefit from the integration of CORBA and Web technologies.

In the first phase of the DCP prototype development the main emphasis was on 'proof-of-concept', i.e. to prototype the integration of Web, Java applets, CORBA objects with a legacy service. In the next phase of the project such issues as security, fault-tolerance, multi-threading, and support of real-time processing will be considered.

Several papers [20], [21], [22], [23], [24], [25], [26], [27] have treated CORBA/OSI management integration with encouraging conclusions, but also bringing forth the drawbacks involved.

Notwithstanding, it seems that the next generation commercial network management platforms will support native CORBA communication [28]. In our case it means that the DCP prototype objects can communicate directly with services included into e.g. such systems as HP's future Synergy technology [29] based platform.

During the project it has become evident, that even though current commercial ORBs are not optimized [30], the CORBA technology is mature enough to be used as a base for the telecom software platform development. 
The different architecture of open distributed telecom systems, as opposed to monolithic systems, still poses the main difficulty. Even though we have found the learning curve of CORBA usage moderate, the combination of Web/Java/CORBA requires patient learning. The emerging Java-ORB technology has the potential to make life easier. We have also found the use of object databases very useful in conjunction with the adoption of object-oriented technology such as Java and CORBA.

\section{REFERENCES}

[1] Object Management Group, The Common Object Request Broker: Architecture and Specification, Revision 2.0, July 1995.

[2] ITU-T M.3010, Principles for a Telecommunications Management Network, Draft June 1995.

[3] A Simple Network Management Protocol, RFC 1157, May 1990.

[4] ISO 10040, Information technology - Open Systems Interconnection - Systems management overview, November 1992.

[5] ISO 9595, Information technology - Open Systems Interconnection - Common management information service definition.

[6] ISO 9596, Information technology - Open Systems Interconnection - Common Management Information Protocol.

[7] Black, U., Network Management Standards, The OSI, SNMP and CMOL Protocols, McGraw-Hill, 1992.

[8] Object Management Group, Common Facilities Architecture, Revision 4.0, November 1995.

[9] Object Management Group, CORBAServices: Common Object Services Specification, March 1996.

[10] ISO/IEC 10164-16, Information Technology - Open Systems Interconnections Systems Management - Management Knowledge Management Function.

[11] IBM Corp., CMIP Run!, Vol. 2 No. 4, 94.

[12] ISO 9594-1, The Directory - Overview of Concepts, Models, and Services.

[13] Object Management Group, Trading Object Service, RFP 5 Submission, May 1996.

[14] Object Management Group, Object Property Service, OMG TC Document, June 1995.

[15] S. Rahkila, S. Stenberg, Nokia XMP++: An Object-Oriented Approach to TMN Application Development, Proceedings TINA '95, Volume 1, Melbourne February 1995.

[16] X/Open, Management Protocols API (XMP), CAE Specification, Mar 1994.

[17] The ADAPTIVE Communication Environment (ACE), http://www.cs.wustl.edu/ schmidt/ACE.html

[18] ABCL : an object-oriented concurrent system, ed. by Akinori Yonezawa, MIT Press, 1990

[19] J. Reilly, The World-wide Web and Programming Future Broadband Network and Service Management Applications, Presented at the NWPER'96, Aalborg May 1996. http://misa.zurich.ibm.com/Consortium/doc/papers 
[20] Luca Deri, Network Management for the 90s, Submitted to ECOOP96, Linz July 1996. http://misa.zurich.ibm.com/Consortium/doc/papers

[21] Della Torre, C., A Generic Distributed Service Management Test Bed Integrating CORBA and the XMP API, Proceedings ECOOP '94, July 1994.

[22] O'Sullivan, D., CORBA and Telecoms Management - can they live in perfect harmony?, Proceedings DOOC '95, October 1995.

[23] Davis, J., Du, W., Kirshenbaum, E., Moore, K., Robinson, M., Shan, M-C., Shen, F., CORBA Management of Telecommunications Network, Proceedings DOOC '95, October 1995.

[24] Park, J-T., Ha, S-H., Hong, J.W., Song, J-G., Design and Implementation of a CORBA-Based TMN SMK System, Proceedings NOMS ‘96, April 1996.

[25] Dittrich, A., Höft, M., Integration of a TMN-based Management Platform into a CORBA-based Environment, Proceedings NOMS ‘96, April 1996.

[26] Kong, Q., Chen, G., Integrating CORBA and TMN Environments, Proceedings NOMS ‘96, April 1996.

[27] Usländer, T., Brunne, H., Management View upon CORBA Clients and Servers, Proceedings ICDP ‘96, February 1996.

[28] Hewlett-Packard, Press Release, http://hpcc998.external.hp.com:80/nsmd/ov/whatisov/telecom.txt

[29] Herman, J., The Sorry State of Enterprise Management, Distributed Computing Monitor, March 1996.

[30] Gokhale, A., Schmidt, D., Measuring the Performance of Communication Middleware on High-Speed Networks, Submitted to SIGCOMM Conference, ACM, August 1996.

\section{BIOGRAPHY}

Susanne Stenberg, Project Manager, Nokia Research Center (Finland), Distributed Computing Platforms technology area.

Sakari Rahkila, R\&D Manager, Nokia Research Center (Finland), Distributed Computing Platforms technology area. 